\title{
Sammelreferat.
}

\section{Neuere Arbeiten über Bildung der Blütenfarbstoffe.}

Sammelreferat vom Standpunkte der Mendelspaltung.

Von Elisabeth Schiemann.

\section{Literatur.}

1. 1902. Chodat u. Bach I: Über Oxydasen. Chem. Ber. 35. S. 1270, 2466, 394;3.

2. 1909. - II: Über Peroxydase. Chem. Ber. 36. S. 600, 606

3. - III: Über Katalase. Chem. Ber. 36. S. 1756.

4. 1905. Molisch, H.: Uber amorphes und kristallisiertes Anthonyan. Bot. Ztg. S. 14; bis 162 .

5. 1906. Grafe, V.: Studien über das Anthacyan. Sitzungsber. k. Ak. Wien $115 \mathrm{I}$. S. 975 .

6. 1907. Wheldale, M.: Inheritance of flower-colour in Antirrhinum majus. Proc. Roy. Soc. London B 79 p. 288-305.

7. 1908. Palladin, W.: Die Verbreitung der Atmungschromogene bei den Pflanzen. Ber. d. d. Bot. Ges. 26. S. 378 u. 389.

8. Baur, E.: Einige Ergebnisse der experimentellen Vererbungslehre. Beil. med. Klinik IV. S. 265.

9. 1909. Wheldale, M.: The colours and pigments of flowers with special reference to Genetics. Proc. Ray. Soc. London B 81 p. 44-60.

10. Grafe, V.: Studien über das Anthocyan. II. Sitz. k. Ak. Wien 118 I. S. 1033.

11. Palladin, W.: Die Verbreituag der Atmungschromogene III. Ber. d. d. Bot. Ges. 27. S.

12. Combes, R.: Rapport entre les composés hydrocarbonés et la formation de l'antlsocyane. Aun. sc. nat. IX. p. $275-303$.

13. Wheldale, M.: On the nature of Anthocyanin. Proc. Cambr. Phil. Soc. XV. pt. II. p. $137-168$.

14. - Further observations upon the inheritance of flower-colour in Antirhinum majus. Roy. Soc. Rep. Evol. Comm. Rep. V. p. 1-26.

15. - Note on the physiological interpretation of the Mendelian factors for color in plants. Roy. Soc. Rep. Evol. Comm. Rep. V. p. 26-31.

16. 1910. Wheldale, M.: Plant oxydases and the chemical inter-relationships of colour varieties. Progr. rei Bot. III. p. 457-474.

17. Baur, E.: Vererbungs- und Bastardierungsversuche mit Antirrhinum. Ztschr. ind. A bst. u. Vererb. III. S. 34-98. 
18. 1910. Wheldale, M.: Vererbung der Blütenfarbe bei Antirrhinum majus. Ztschr. ind. Abst. u. Vererb. III. S. 321-333.

19. Keeble, F. and Pellew, C.: White flowered varieties of Primula sinensis. Journ. Genetics I. p. $1-5$.

20. Armstrong, H. E. a. Armstrong, E. F.: The origin of osmotic effects. III. Proc. Roy. Soc. London B 82. p. 588-602.

21. Combes, R.: Du rôle de l'oxygène dans la formation et destruction des pigments rouges anthocyaniques chez les végétaux. C. R. Acad. Sc. Paris 150 . p. $1186,1532$.

22. 1911. Combes, R.: Recherches sur la formation des pigments anthocyaniques. C. R. Ac. Sc. Paris 153 . p. $886-889$.

23. Grafe, V.: Studien über das Anthocyan. III. Sitz. k. Ak. Wien 120 II. S. 765.

24. Wheldale, M.: The chemical differentiation of species. Biochem. Journ. V. p. 445 .

25. - On the direct Guajacum reaction given by plant extraets. Proc. Roy. Soc. London B 84. p. 121-124.

26. - On the formation of Anthocyanin. Journ. Gen. I. p. 133-158.

27. Nierenstein, M. u. Wheldale, M.: Beiträge zur Kenntnis der Anthocyane. I. Ber. d. d. Chem. Ges. 44. S. 3487.

28. Gregory: Studies in Primula sinensis. Journ. Gen. p. 73-132.

29. Thoday: Inheritance of the yellow tinge in sweat pea colouring. Proc. Cambr. Phil. Soc. 16. p. $71-84$.

30. Armstrong, H. E. a. Armstrong, E. F.: The function of hormones in regulating metabolism. Ann. Botany 25. p. 507-519.

31. 1912. Keeble, F. and Armstrong, E. F.: The formation of Anthocyan pigments in plants. I: The distribution of oxydases in plants and their rôle in the formation of pigments. Proc. Roy. Soc. London B 85. p. 214-218.

32. - do. II: The oxydases of Cytisus Adami. Ibid. p. 460-465.

33. - do. III: The xôle of oxydases in the formation of Antbocyan pigments of plants. Journ. Genetics II. p. $277-311$.

34. Nierenstein, M.: Beitrag zur Kenntnis der Anthocyane. II. Ber. d. d. Chem. Ges. 45. S. $499-501$.

35. Chodat, R.: Nouvelles recherches sur les ferments oxydatifs. Arch. Sc. Phys. Nat. 33. p. 70, 225.

36. 1913. Keeble, F., Armstrong, E. F. and Jones, W. N.: The formation of Anthocyan pigments IV: The chromogens. Proc. R. Soc. London B 86. p. $308-317$.

37. Jones, W. N.: The formation of Anthocyan pigments V: The clromogens of white flowers. Proc. Roy. Soc. London B. 86. p. 318-323.

38. Keeble, Armstrong and Jones: The formation of Anth. pigments. VI: Proc. Roy. Soc. London B 87. p. 113-131.

39. Wheldale, M.: The flower pigments of Antirrhinum majus. I: Method of preparation. Biochem. Journ. VII, p. 87-91.

40. Wheldale, M. and Bassett, H. L.: do. II: The pale yellow or ivory pigment. Biochem. Journ. VII. p. 441-444.

41. Combes, R.: Production expérimentale d'une anthocyanine. C. R, Ac. Sc. Paris 157. p. 1002-1004. 
42. 1913. Combes, R.: Passage d'un pigment anthocyanique extrait des ferilles rouges d'automne au pigment jaune contenu dans les feuilles vertes de la même plante. C. R. Ac. Sc. Paris 157. p. 14õ4-1457.

43. - Untersuchungen über den chemischen Prozeß der Bildung der Anthocyane. Ber. d. d. Bot. Ges. 31. S. 570-578.

44. Tswett, M.: Beitrag zur Kenntnis der Anthocyane. Biochem. Ztschr. 58. S: $225-235$.

45. Peche, K.: Über eine Gerbstoffreaktion und ihre Beziehnngen zu den Anthocyanen. Ber. d. d. Bot. Ges. 31. S. $462-471$.

46. Willstätter, R. u. Everest, A. E.: Untersuchungen über die Anthocyane. I: Über die Farbstoffe der Kornblume. Liebigs Ann. Chem. 401. S. $189-232$.

47. Wheldale, M. and Bassett, H. L.: The chemical interpretation of Mendelian factors for flower colour. Proc. R. Soc. London B 87. p. 300-311.

48. 1914. Tswett, M.: Zur Kenntnis des vegetabilischen Chamaeleons. Ber. d. d. Bot. Ges. 32 . S. $61-68$.

49. Willstätter, R.: Über die Farbstoffe der Blüten u. Früchte. Sitzungsber. Kgl. Akad. Wiss. Berlin Phys. math. Kl. XII. S. 402-411.

50. Wheldale, M. and Bassett, H. L.: The flower pigments of Antirrhinum majus III: The red and magenta pigments. Biochem Journ. VIII. p. $204-208$.

51. Everest, A. E.; The production of Anthocyanins and Anthocyanidins. Proc. Roy. Soc. London B 87. p. 444.

52. Willstätter, R. u. Mallison, H.: Über die Verwandtschaft der Anthocyane und Flavone. Sitzungsber. Kgl, Akad. Wiss. Berlin Phys. math. Kl. XXIX. S. $769-777$.

53. Wheldale, M. and Bassett, H. L.: On a supposed synthesis of Anthocyanin. Journ. Genetics IV. p. 103-108.

Es liegt eine Reihe neuerer Arbeiten vor, die das Ziel haben, die Natur und Bildungsweise der Blütenfarbstoffe kennen zu lernen, um den durch die Bastardierungsarbeit festgestellten sog. Mendelfaktoren eine materielle Grundlage geben zu können.

Seit langer Zeit ist diesen Forschungen eine bestimmte Richtung gewiesen dadurch, daß man die Bildung der Anthocyane als einen Oxydationsprozeß auffaßte. Vor allem andern hat die Erklärung der Mendelfaktoren bei der Vererbung der Blütenfärbe in der: sorgfältig durchgearbeiteten Theorie von Miss Wheldale auf der Oxydationshypothese gefußt. Ihr sind Keeble und seine Mitarbeiter gefolgt.

Da mußte es denn einigermaßen Aufsehen erregen, als im Jahre 1913 R. Combes $(41-43)$ auf Grund spezieller chemischer Untersuchungen die Ansicht aussprach, daß3 es sich im Gegenteil bei der Bildung der Anthocyane aus dem postulierten Leukokörper um einen Reduktionsvorgang handle. Ebenso ist auch von seiten Keebles (38) ein Vorstoß gegen die volle Gültigkeit der Wheldaleschen Hypothese ausgegangen. 
Darauf trat Tswett (44, 48) mit der Herstellung eines künstlichen Anthocyans hervor; vor allem aber ist es dem Darsteller des Chlorophylls, Willstätter $(46,49,52)$ und seinen Mitarbeitern gelungen, natïrliches Anthocyan rein darzustellen und seine Konstitution zu ermitteln und damit ein Problem zu lösen, an dem seit über 60 Jahren von verschiedenen Seiten vergeblich gearbeitet ist. Gleichzeitig gelang Miss Wheldale $(40,47)$ die reine Darstellung erst der gelben, in jüngster Zeit auch der rot-violetten Farbstoffe von Antirrhinum (51). Während man so einerseits beginnt, festen Boden unter die Füße zu bekommen, ist andererseits die Mendelsche Faktorenlehre auf dem Gebiete der Blütenfärbung dadurch an einen Wendepunkt geführt. Denn es liegt ihr nunmehr die Aufgabe ob, die Ergebnisse der Bastardforschung, deren Erklärung in alter Weise nicht mehr Stand hält, mit dem neuen Tatsachenmaterial, das von chemischer Seite geliefert ist, zu vereinigen. Es ist deshalb vielleicht von Interesse, das auf Grund der Wheldaleschen Hypothese bisher Erreichte zu präzisieren, die für unser Problem in Betracht kommenden neuen Ergebnisse der Chemie kurz zusammenzustellen und die Fragen, die jetzt erneuter Durcharbeit bedürfen, hervorzuheben.

Durch die Bastardierungsarbeiten von Baur, Wheldale, Bateson, Saunders, Keeble, Gregory, Shull u. a. ist für die Bestimmung der Blütenfarben übereinstimmend eine Anzahl von Faktoren herausgearbeitet worden, von denen die wichtigsten hier noch einmal kurz zusammengefaßt werden sollen und zwar:

I. für Antirrhinum als Vertreter einer Pflanzengruppe, die gelbe und ròt-blaue Farbstoffe im Zellsaft gelöst enthält.

$\mathrm{B}=$ Grundfaktor für Farbe überhaupt; $\mathrm{BB}$ od. $\mathrm{Bb}$ ohne die übrigen Farbfaktoren $=$ gelb $; \mathrm{bb}=$ weiß;

$\mathrm{C}=$ wandelt in Gegenwart von $\mathrm{B}$ gelb in elfenbein.

$\mathrm{F}, \mathrm{R}, \mathrm{M}, \mathrm{L}=$ Faktoren, die jeder nur bei Anwesenheit der vorhergehenden (bis zu B rückwärts) verschiedene Abstufungen von rot hervorbringen und zwar bei Gegenwart von $\mathrm{C}$ auf elfenbein-Grund, bei Abwesenheit von $\mathrm{C}$ auf gelbem Grund ${ }^{1}$ ).

II. für Lathyrus als Vertreter einer Pflanzengruppe, wo die gelben Zellsaftpigmente fehlen ${ }^{2}$ ).

$\mathrm{F}$ u. $\mathrm{R}=$ Grundfaktoren für Farbe überhaupt - von denen jeder für sich wirkungslos ist, beide zusammen dagegen rot ergeben, also $F r$ oder $f R$ oder $\mathrm{fr}=$ weiß.

$\mathrm{B}=$ bläuender Faktor, nur wirksam mit $\mathrm{F}+\mathrm{R}$, wandelt rot in purpur.

1) So die Bezeichnung bei Baur I. Aufl.; in der II. Aufl., deren Erscheinen aber nach Abschluß der hier besprochenen Arbeiten fällt, ist die Bezeichnung etwas anders; ebenso Matthiola.

2) Bez. n. Bateson, Mendels Princ, dentsche Ausgabe. S. 89. 
Die Hypothese, die Miss Wheldale mit Benutzung der Bach-Chodatschen Theorie über Oxydasenwirkung zur Erklärung dieser großen Anzahl von Mendelfaktoren für die Blütenfärbung aufgestellt hat und die Ref. in Band IX 1913, S. 277 skizziert hat, läßt sich in die nachfolgenden Gleichungen fassen:

$$
\begin{aligned}
& \text { A. Glukosid }+\mathrm{H}_{2} \mathrm{O} \underset{\text { Glukosid- }}{-\ldots} \text { Chromogen }+ \text { Zucker } \\
& \text { B. Chromogen }+\mathrm{O}_{2} \underset{\text { oxydase }}{\longrightarrow} \text { Anthocyan }
\end{aligned}
$$

Danach ist die Bedingung für die Färbung (Gleichung B) das Vorhandensein des Chromogens. Liegt dieses in der Blüte fertig vor, so verfügt die Pflanze über einen Faktor für Blütenfarbe (Beispiel: Antirrhinum). Ist es aber als Glukosid nicht reaktionsfähig, so muß als zweiter Grundfaktor noch ein glukosidspaltendes Enzym vorhanden sein; in diesem Fall wird der erste Prozeß der Farbbildung durch Gleichung A ausgedrückt (Beispiel: Lathyrus).

Die Faktoren, welche die verschiedenen Nuancen und Zeichnungen bedingen $(\mathrm{F} \rightarrow \mathrm{R} \rightarrow \mathrm{M} \rightarrow \mathrm{L}$ fiur Antirrhinum nach Baur), legt Miss Wheldale als verschiedene spezifisch wirkende Oxydasen aus $(14,15)$. Die dunkleren Farben sollen dabei eine höhere Oxydationsstufe der helleren sein. Fehlt eine Oxydase, so wird der Prozeß an der betreffenden Stelle abgebrochen, wenn auch Oxydasen für weitere Oxydationen noch vorhanden sein sollten (z. B. $\mathrm{L}=$ schwärzender Faktor, nicht wirksam ohne $\mathrm{F}$ oder $\mathrm{M}$; vgl. S. 86).

Auf dem Fehlen der Grundfaktoren beruht die Existenz rein weißer Rassen. Es ist klar, daß sie sehr verschiedene Erbformeln besitzen können, je nach der Kombination der vorhandenen, aber ohne den Grundfaktor wirkungslosen farbbildenden Faktoren; so kennt Baur weiße Sippen von Antirrhinum von der Formel: bbFFcc neben solchen von der Formel: bbffec.

Die Kreuzungsresultate zweier weißer Rassen sind von der Zahl der Grundfaktoren für Farbe abhängig; theoretisch kann aus der Kreuzung dieser rezessiv weißen Antirrhinum nie eine farbige Deszendenz hervorgehen; sie ist in der Tat auch nicht beobachtet, während die Kreuzung von elfenbein mit weiß verschiedener Konstitution verschiedene Nachkommənschaft ergab (Beispiel: Baur, Vererbungslehre, S. 70. II. Aufl., S. 95).

Anders bei Pflanzen mit zwei Grundfaktoren für Farbe ( $F$ und $R$ ), wie Lathyrus. Hier muß eine Kreuzung von weiß von verschiedener Formel bei geeigneter Kombination eine farbige Deszendenz haben ( $\mathrm{Fr} \times \mathrm{fR}=\mathrm{FfRr}$ ). Auch diese Voraussetzung ist experimentell bestätigt worden (Bateson u. Punnett, Journ. Gen. I, Wheldale 15, p. 31).

Neben den rezessiv weißen Sippen steht nun eine zweite Gruppe, die der dominant weißen, die bei Kreuzung mit farbigen Sippen eine weiße $F_{1}$ 
mit Aufspaltung in $\mathrm{F}_{2}$ i. V. 3 weiß: 1 farbig geben. Beispiele und Nachweise werden den Lesern der Zeitschrift hinlänglich bekannt sein.

Zur Erklärung eines solchen Verhaltens ist die Annahme gemacht, daß in diesen Sippen der Grundfaktor für Farbe zwar vorhanden, aber durch einen Hemmungsfaktor (inhibitor) in seiner Wirksamkeit gehindert ist. Nach Gleichung $\mathrm{A}$ und $\mathrm{B}$ kann dieser Faktor sowohl die Spaltung des Glukosides als die Oxydation des Chromogens verhindern.

Um diese Hypothesen zu verifizieren haben Miss Wheldale einerseits, Keeble und Armstrong andererseits den chemischen Nachweis der postulierten Substanzen zu führen gesucht. Arbeiten, die andere Ziele verfolgten, reihen sich mit ihren Ergebnissen ergänzend ein. Es sei aber gleich voraus bemerkt, daß aus dem Vorhandensein der Substanzen ihre Mitwirkung in den Farbbildungsprozessen zwar möglicherweise bzw. wahrscheinlich, aber nicht notwendig folgt.

\section{Das glukosidspaltende Enzyn.}

Der Nachweis eines glukosidspaltenden Enzyms gelang für eine große Anzahl anthocyanhaltiger Pflanzen. Der Besitz solchen Enzyms ist jedenfalls bei den Pflanzen eine häufige Erscheinung (Wheldale 26), beschränkt sjch aber nicht auf die anthocyanhaltigen.

\section{Die Oxydasen.}

Ebenso sind Oxydasen weit verbreitet. Da die hierfür in Betracht kommenden Arbeiten von Keeble und Armstrong $(31,33)$ in dieser Zeitschrift schon ausfïhrlich besprochen sind (vgl. Ref. Bd. X, 1913, S. 277) ${ }^{1}$ ), so sollen hier nur die wichtigsten Punkte hervorgehoben werden:

1. Direkte Oxydasen (d. h. das System Peroxyd + Peroxydase) konnten in allen Pflanzen, die Anthocyan enthalten, nachgewiesen werden.

2. Die Verbreitung der Oxydasen ist jedoch weit größer als die der Anthocyane. Sie finden sich nicht nur in anthocyanfreien Pflanzen an Stellen, die bei verwandten Sippen Anthocyan führen, sondern auch ganz unabhängig von der Farbverteilung. Sie müssen also in diesen Geweben eine andere Funktion haben.

3. Die Peroxydasen allein sind noch weiter verbreitet, als die direkten Oxydasen, d. h. nicht überall, wo die Oxydasereaktion positiv verläuft, ist auch das organische Peroxyd nachzuweisen. Ist es zur Farbbildung notwendig, so muß die Annahme gemacht werden, die z. B. Chodat vertritt, daß es ad hoc je nach Gebrauch, etwa mit Hilfe des Luftsauerstoffs gebildet wird. Es muß zugegeben werden, daß diese Erwägungen die Oxydationshypothese nicht zwingend bestätigen.

1) vgl. Anm. 1 S. 88 . 
4. Anderseits fand Miss Wheldale $(14,15)$ die Verteilung der Oxydasen der der Anthocyane nicht nur parallel gehend, sondern auch die Stärke der Realition der Intensität der Färbung proportional. Auf diesen Nachweis gründete sich ihre Annahme, daß innerhalb einer Farbreihe die dunklere Nuance aus der helleren durch eine weitere Oxydation vernittels einer spezifischen Oxydase vor sich geht. Jede dieser Oxydasen stelle einen Mendelfaktor dar. Daher die Konstanz bei der Vererbung der Abstufung dunkel - mittel - hell einer bestimmten Farbe (vgl. dagegen unten S. 91).

\section{Der Hemmungsfaktor.}

Als Hemmungsfaktor bei dominant weißen Blüten wurde ein oxydationshemmendes Enzym angenommen; ein solches konnten Keeble und Armstrong (33) in den dominant weißen Blüten von Primula sinensis nachweisen. Genauere Angaben über diesen Hemmungsfalktor fehlen dort aber noch.

An diesem Punkt setzten die Arbeiten des letzten Jahres von Keeble, Armstrong und Jones, Wheldale und Bassett ein (36-40).

Es ist eine alte Beobachtung, daß anthocyanhaltige Blüten durch absoluten Alkohol entfärbt werden, daß aber die Farbe der Petalen wie der Lösung nach einiger Zeit wiederkehrt, besonders wenn man Wasser zusetzt.

Keeble, Armstrong und Jones (36; Objelit ist Matthiola) sehen diese Regeneration - wie es ja bisher meist geschah - als einen Oxydationsvorgang an. Für diese Auffassung führen sie die Tatsachen an, daß Zusatz von $\mathrm{H}_{2} \mathrm{O}_{2}$ die Regeneration der blauen wie der roten Farbe beschleunigt, während sie in ausgekochtem Wasser nicht stattfindet, durch HCN verhindert wird (HCN ist ein Enzymgift) und bei hoher Temperatur schneller erfolgt. Ist aber die Regeneration des Farbstoffs eine Oxydation, so liegt es nahe, die Wirkung des Alkohols in der Freimachung eines reduzierenden Agens zu sehen und zwar als eine Folge seiner wasserentziehenden Figenschaft.

Nach dieser Theorie ist in vivo die Wirkung der reduzierenden Substanz abhängig von der Menge des in der Zelle enthaitenen Wassers.

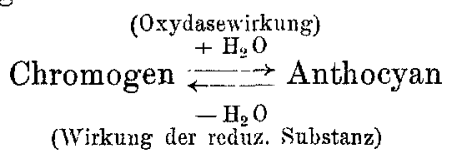

Will man mit den Verff. in den dominant weißen Blüten diese Art von „Hemmung“ annehmen, so würde der "Faktor" einen Stoff bedeuten, der der Zelle Wasser entzieht.

Nun ist einmal der Nachweis dieser reduzierenden Substanz nur für farbige Blüten geführt, also gerade für einen Fall, in dem er der Theorie nach wegen zu hohen Wassergehaltes unwirksam ist. Dann aber haben Wheldale und Bassett (47), kürzlich auch Tswett (44) gegen die Erklärung Bedenken erhoben. 
Es zeigte sich nämlich, daß der Wassergehalt nicht entscheidend ist und die Regeneration der Farbe in der alkoholischen Lösung weder ein Oxydations- noch ein Reduktionsvorgang sein kann. Die Möglichkeit, daß es sich um einen Vorgang vom Typus der Indikatoren handelt, haben K., A. und J. selbst von der Hand gewiesen. Wheldale und Bassett äußern über die sich abspielenden Vorgänge Mutmaßungen, die dem wahren Sachverhalt nahe kommen, der endgiiltig von Willstätter (49) aufgedeckt worden ist. Danach „handelt es sich bei dieser Erscheinung um Isomerisation, welche der Umwandlung eines Triphenylmethanfarbstoffes in sein Karbinol entspricht. Die Entfärbung läßt sich durch Zusatz gewisser Salze ( $\mathrm{NaCl}$, $\mathrm{NaNO}_{3}$ ) verzögern und mit überschüssiger Säure ganz verhindern". Daß daneben auch reduzierende Substanzen in den Blüten nachweisbar sind, wird damit nicht bestritten; sie müssen aber andere physiologische Funktionen haben.

Es ist nun (Jones 37) noch eine andere Möglichkeit für das Zustandekommen weißer Blïten und zwar rezessiver, die sowohl aktive Oxydase als oxydationsfähiges Chromogen enthalten, ausgesprochen worden, nämlich die, daß Chromogen und Oxydase in der Pflanze räumlich getrennt sind, so daß sie nicht miteinander reagieren können. Verf. denkt sie sich in verschiedenen Zellen oder verschiedenen Teilen einer Zelle lokalisiert. Dieser Fall von Albinismus liege bei Lychnis coronaria var. alba vor; er reiht sich an die Beobachtungen der vorigen Arbeit an.

Aus den Petalen dieser Blïten konnte Jones einmal eine oxydasefreie Chromogenlösung (A), zweitens eine chromogenfreie oxydasehaltige Lösung (B) herstellen; beim Zusammenbringen beider Lösungen $(A+B)$ resultiert ein rötlichbraunes Pigment. Das Chromogen der Blïte kann also durch die eigene Peroxydase gebräunt werden, wenn die räumliche Trennung der beiden Stoffe aufgehoben wird; dies geschieht durch Veränderung der Permeabilität des Plasmaschlauchs, hier durch Alkohol.

H. E. u. E. F. Armstrong haben auf Stoffe, die die Permeabilität des Protoplasten verändern können, indem sie andern Stoffen gewissermaßen den Weg zwischen den Molekülen des Plasmaschlauchs bahnen, den Ausdruck "Hormone" ${ }^{1}$ ) übertragen. $\mathrm{CO}_{2}, \mathrm{HCN}$ und Phenole, die im Stoffwechsel der Pflanzen ja häufig auftreten, können die Rolle solcher Hormone spielen. Die Unfähigkeit sie zu bilden wäre dann das Charakteristikum weißer Rassen, in denen sich Chromogen und Oxydase nachweisen lassen. Es fragt sich indessen, ob man diese rotbräunliche Färbung, die den postmortalen Pig-

1) Starling bezeichnet als Hormone chemische Stoffe, die, von bestimmten Zellen abgeschieden, Reize weiterleiten, die alsdann an entfernten Stellen eine Reaktion hervorrufen. Ebenso stimulieren eine große Reihe - stets anhydrophiler - Stoffe, die in die Zelle einzudringen vermögen, in dieser die Enzymtätigkeit; A. nennen sie daher Hormone (Ann. Botany 25, p. 510). 
menten gleicht, überhaupt für die Bildung der Anthocyane als Analogon heranziehen darf.

Als Hemmungsfaktor sind von Keeble und Armstrong (38) endlich auch die plastischen Pigmente angesprochen worden, die z. B. die gelbe Farbe von Cheirantus Cheiri bedingen, und die verschwinden in dem Maße, als die dunklen Anthocyane auftreten. Doch läßt sich aus den wenigen Daten noch nichts Sicheres erkennen, auch kommen bei Matthiola nach E. R. Saunders alle Anthocyanfarben sowohl auf weißem Grunde als auf der durch Chromoplasten hervorgerufenen Crèmefarbe vor (vgl. Bateson, Mend. Princ., S. 93).

Auf Grund der Oxydationshypothese sind also bisher folgende Typen von weißen Blüten unterschieden worden:

A. Rezessiv weiße - durch Verlust eines Faktors aus Gleichung B.

Es sind vorhanden 1. Oxydase + Chromogen, aber räumlich getrennt (Beispiel: Jones, Anemone, Lychnis, Chrysanthemum); die farbige Varietät besitzt einen Faktor zur Bildung eines Hormones; der weißen Varietät fehlt der Faktor für Hormone.

2. Peroxydase + Chromogen; es fehlt der Faktor zur Bildung des organischen Peroxyds (Beispiel: Keeble u. Armstrong, Dianthus barbatus - Sweet William, Dianthus Caryophyllus - Mrs. Sinkins).

3. Peroxydase, kein Chromogen (Beispiel: Flumbago capensis, Suainsonia Tacsonia - Primula sinensis ${ }^{1}$ ), Pisum sativn, Lathyms odoratus, Lychnis; Jones, Keeble u. Armstrong, Shull u. a.).

B. Dominant weife mit Hemmungsfaktor, Oxydase inaktiv.

1. Reduzierende Substanz vom Enzymtypus (Beispiel: Primula sin.; Keeble u. Armstrong).

2. Reduzierende Substanz bei geringem Wassergehalt der Zelle (kein weißblühendes Beispiel, Matthiola incana; Keeble, Armstrong und Jones [die Existenz dieses Typus ist sehr fraglich]).

3. Plastische Pigmente (Cheiranthus Cheiri; Keeble u. Armstrong).

\section{Die Chromogene.}

Ich komme nun auf die Farbstoffe selbst und damit auf ein zurzeit aussichtsvolleres Gebiet.

Bekanntlich werden die Pflanzenfarbstoffe eingeteilt in plastische und im Zellsaft gelöste.

1. Die plastischen Pigmente sind Chlorophyll (enthaltend $\mathrm{C}, \mathrm{O}$, $\mathrm{H}, \mathrm{N}, \mathrm{Mg}$ ), Carotin (C, H) und Xanthophyll (C, H, O).

1) In dem auf diese Arbeiten bezüglichen Referat (ds. Zeitschr. X, S. 279) sind bei der Zusammenfassung die beiden Worte Chromogen und Oxydase (Zeile 16) vertauscht worden. 
2. Die Zells aftpigmente zerfallen in zwei Gruppen:

a) gelbe, ätherlösliche Stoffe aus der Gruppe der Flavone, die von Perkin in großer Zahl untersucht worden sind. Willstätter schlägt vor, für diese den schon 1835 von Marquart eingeführten Namen Anthoxanthine beizubehalten, der seither oft, aber nicht scharf umgrenzt, gebraucht worden ist.

b) blau-violett-rote in Alkohol lösliche, in Äther unlösliche Stoffe, die sog. Anthocyane, von Willstätter als Anthocyanine bezeichnet.

\section{a) Die Anthoxanthine.}

Die Anthoxanthine sind meist als Glukoside in der Pflanze enthalten und als solche sehr verbreitet, treten jedoch seltener als Blütenfarbstoffe auf, als in den vegetativen Teilen der Pflanzen, den Stengeln, Rhizomen, Blättern. Einzelne, z. B. Quercetin, kommen auch frei vor. Es sind aromatische Substanzen mit phenyliertem Pyronkern.<smiles>CC1COCCC1=O</smiles>

Pyron<smiles>CC1COc2ccccc2C1</smiles>

$\mathrm{CO}$

Pheno- $\gamma$-Pyron

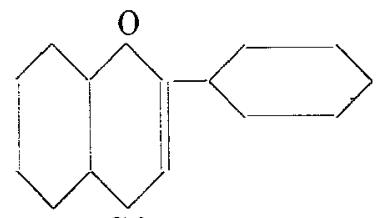

$\mathrm{CO}$

[Chromon ${ }^{1}$ )]

Flavon

$\left[\right.$ Phenylchromon $\left.\left.^{1}\right)\right]$

Neuerdings hat Miss Wheldale (40) aus den elfenbeinfarbigen und gelben Blüten von Antirrhinum die Farbstoffe rein dargestellt (vgl. Fig. 2 u. 3 in Baur, Vererbungslehre, Taf. I u. Baur, diese Zeitschr. IIT, Taf. I). Es enthalten die Blüten von Fig. 3 in allen Schichten, die von Fig. 2 in den inneren Geweben den elfenbein-Farbstoff, Fig. 2 in der Epidermis der Lippen den gelben Farbstoff.

Der elfenbein-Farbstoff konnte als Apigenin identifiziert werden,<smiles>O=C1CC(C2CCC(O)CC2)OC2CC(O)CC(O)C1C2</smiles>

ein aus dem Glukosid Apiin in Apium Petroselinum gewonnenes Hydroxyflavon (Über die chemischen Einzelheiten s. Original), der gelbe Farbstoff ist Luteolin (47), das in Genista tinctoria, Digitalis und Resela luteola vorkommt, in letzter Pflanze, was ganz interessant ist, mit Apigenin gemeinsam.

1) Nach Euler. 
Luteolin ist von Apigenin durch den Mehrbesitz einer Hydroxylgruppe unterschieden.

Interessant ist, daß die ersten Farbstoffniederschläge, die aus den Oberund Unterlippen der Blüten hergestellt waren, Schmelzpunkte zwischen 250 bis 338 schwankend ergaben. Nun besitzt die Unterlippe aller AntirrhinumBlüten mit Ausnahme der weißen einen gelben Flecken, der sich ganz unabhängig von der gelben Farbe im übrigen vererbt. Verff. ließen deshalb zur Untersuchung der gelben Farbstoffe die Unterlippe fort; exst jetzt ergab sich ein fester Schmelzpunkt bei $324^{\circ}$. Die für das Auge gleichartigen, aber genetisch verschiedenartigen Farbstoffe sind also auch chemisch verschieden.

Was zieht nun Miss Wheldale aus diesen Ergebnissen für einen Schluß auf die Erbfaktoren?

Es war BBec die Formel der gelben, BBCC oder BbCC die der elfenbeinfarbigen Blüten. Es entsteht also gelb aus elfenbein durch Verlust des Faktors C. Anderseits stehen Apigenin und Luteolin nach ihrer empirischen Formel im Verhältnis:

$$
\text { Luteolin }=\text { Apigenin }+(\mathrm{OH}) \text {. }
$$

Miss Wheldale weist selbst darauf hin, daß mit der Annahme, der Faktor $\mathrm{C}$ bedeute ein Enzym, das die Addition der Hydroxylgruppe reguliert, nichts gewonnen sei. Auch ist nicht zu ersehen, warum $\mathrm{C}$ epistatisch gegenüber $B$ ist. Sie nimmt vielmehr an, daß die Ausbildung der verschiedenen Flavone der Ausdruck eines fundamentalen Unterschiedes in der Struktur der beiden Sippen ist. Dafür spricht auch die Tatsache, daß alle Farben entweder auf elfenbein oder auf gelbem Grunde vorkommen. Möglicherweise ist diese Strukturverschiedenheit begründet auf dem Besitz verschiedener Hydroxybenzoësäuren, aus denen dann verschiedene Flavone aufgebaut werden. Versuche, Flavone aus den weißen Varietäten zu gewinnen, blieben erfolglos. $\mathrm{Zu}$ einer Aufklärung über die materielle Grundlage der Mendelfaktoren hat also die genauere chemische Kenntnis dieser gelben Farbstoffe zunächst nicht gefïhrt.

$$
\text { b) Anthocyanine }{ }^{1} \text {. }
$$

Die eben behandelten Anthoxanthine und ihnen verwandte Hydroxyflavone sollen nun die Chromogene darstellen, aus denen die Anthocyane hervorgehen (vgl. Gleichung B, S. 84). Beide Gruppen von Farbstoffen kommen als Glukoside vor. Dieser Tatsache Rechnung tragend, hat die Hypothese von Miss Wheldale, der zufolge das Chromogen nur in zuckerfreiem Zustand oxydabel ist, folgende Form angenommen: Durch spezifische Glukosidspalter werden aus den Flavonen verschiedene Zucker abgespalten

1) Im folgenden soll der Übereinstimmung mit den zitierten Autoren wegen bis zur Erörterung der Willstätterschen Arbeiten der alte Ausdruck Anthocyane beibehalten werden. 
und dadurch verschiedene Chromogene zur Oxydation freigemacht. Durch Oxydation und Kondensation zu größeren Komplexen entstehen die verschiedenen Anthocyane. Die Gleichung S. 84 geht dadurch in die folgende über:

$$
\begin{gathered}
\text { Glukosid }+\mathrm{H}_{2} \mathrm{O} \longrightarrow \text { (Flavon od.Xanthon) } \\
\quad \mathrm{C} \text { (Chromogen) }+ \text { Sucker } \\
\quad \longrightarrow \text { Sauerstoff } \longrightarrow \text { Anthocyanin. }
\end{gathered}
$$

Die restierenden Hydroxylgruppen werden weiterhin durch Zucker ersetzt, so daß die Anthocyane selbst wieder als Glukoside in der Pflanze vorkommen. Während die verschiedenen Farbtön e durch verschiedene Spaltungen zustande kommen, stellen die Farbintensitäten verschiedene Oxydationsstufen dar. Gegen die Theorie der spezifischen Glukosidspalter wendet sich die letzte Arbeit von Keeble, Armstrong u. Jones. Einmal setze sie mehr Zucker voraus, als in den mit Flavonen auftretenden Glukosiden enthalten sind (nämlich nur zwei), dann aber sprechen die Enzymstudien Chodats gegen sie. Es soll nach ihrer Meinung nicht ein Glukosid vorhanden sein, das verschiedenartig gespalten wird, sondern verschiedene Glukoside, aber nur ein glukosidspaltendes Enzym. Gestützt wird diese These durch die Angabe Perkins, daß die Flavone in den Pflanzen 'meist in Gruppen vorhanden sind.

An Stelle der graduellen Oxydation setzen Verff. das Eingreifen spezifischer Körper in den Gang der Reaktion von Oxydase auf Chromogen. Es entstehen in dieser Weise zwei Gruppen von Farbstoffen:

1. Oxydationsprodukte von Phenolen unter Reaktion mit Aminosüuren - also stickstoffhaltige Produkte.

2. Oxydationsprodukte von Phenolen, hervorgebracht durch organische Sauerstoffüberträger, die den Sauerstoff der niederen Farbstufen zur Bildung der höheren freimachen und die niederen dadurch zu farblosen Substanzen reduzieren.

Die Anregung zu diesen Arbeiten gaben zwei Mitteilungen von Chodat. Er beobachtete einerseits, daß bei Einwirkung pflanzlicher Oxydase auf Glykokoll Kohlensäure, Formaldehyd und Ammoniak gebildet werden; anderseits, daß p-Kresol bei Gegenwart von Oxydase und einer Aminoverbindung eine Serie farbiger Oxydationsprodukte liefert, deren Farbe von der Aminoverbindung abhängt - m. a. W. er beobachtete das Eingreifen von N-Verbindungen in den Verlauf der Farbbildung. Ähnliche Verhältnisse sind aber in der Pflanze leicht realisiert; danach geht dort die Farbbildung folgendermaßen vor sich:

Bei Gegenwart von einem Glukosid (z. B. Arbutin) und seinen Spaltungsprodukten (Phenolen), Spaltungsprodukten der Eiweißkörper $\left(\mathrm{NH}_{3}\right.$, Aminosäuren) und Oxydasen tritt eine Färbung auf, die bestimmt ist durch die verschiedenen reagierenden Substanzen nach dem Schema:

a) Oxydation des Phenols zu Chinon 
b) Reaktion des Chinons mit $\mathrm{NH}_{3}$ in Gegenwart des Phenols, wobei ein farbiger Körper entsteht.

Verff. haben experimentell nach diesem Schema verschiedene Farbserien herstellen können und fanden die Farbe im Gegensatz zu Chodat abhängig von der Natur der Phenole (Arbutin liefert eine andere Farbreihe als Salicin), während verschiedene Stickstoffverbindungen mit dem gleichen Phenol die verschiedenen Töne ein er Farbreihe gaben. Damit ist die Theorie gestützt, daß ein Glukosid nach seiner Spaltung durch ein Enzym infolge von Verbindung mit verschiedenen spezifischen Aminokörpern die betreffende Farbserie gibt.

Die 2. Reihe von Farbstoffen nach Keeble, Armstrong u. Jones sind die, bei denen die eingreifenden Substanzen als Sauerstoffüberträger funktionieren.

Ausgangspunkt war die Beobachtung, daß die Benzidin-Reaktion (Blaufärbung) mit $\mathrm{H}_{2} \mathrm{O}_{2}$ und einer Oxydase bei Zusatz eines weiteren Phenols einen anderen Farbton gibt. Unter den meist gefärbten Oxydationsprodukten der Phenole steht als farbloser Stoff das Methylhydrochinon. Fügt man zu Benzidinoxydase $+\mathrm{H}_{2} \mathrm{O}_{2}+$ Methylhydrochinon, so wird der Sauerstoff, der bei der Blaufärbung vom Benzidin festgehalten wird, jetzt weiter auf das Methylhydrochinon übertragen und liefert mit diesem ein rotes Produkt. Vergleicht man diese Vorgänge mit den bei Vererbung der Blütenfarbe beobachteten, so kommt man zu folgenden zwei sich entsprechenden Schemata,
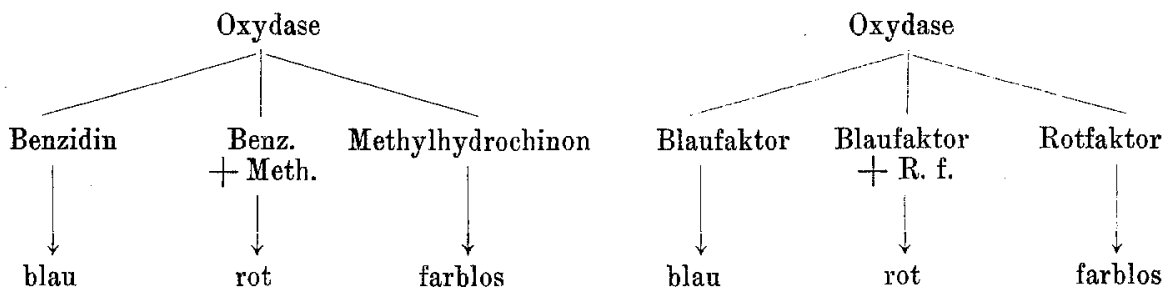

Bei der Rotfärbung funktioniert der Blaufaktor nur als Übertrager des Sauerstoffs auf den ohnedas nicht farbbildenden, etwa phenolartigen Körper, den der Rotfaktor darstellt. Da er dabei selber reduziert wird, so folgt: rot ist völlig dominant über blau (Beispiel: Primula sin.; bei Lathyrus verhalten sich die Farben umgekehrt!).

Damit kommen Verff. zu dem Schluß: Die Faktoren der Mendelspaltung für die einzelnen Farbennuancen sind nicht spezifische Glukosidspalter und spezifische Oxydasen, sondern ein Glukosidspalter, eine Oxydase und eine Anzahl spezifischer Spaltprodukte der Glukoside und Eiweißkörper.

Gegen diese Erörterungen ist vor allen Dingen einzuwenden, daß die Anthocyane stickstofffreie Verbindungen sind. Es bliebe also zunächst der Nachweis zu führen, daß die erzielten Farbreaktionen sich nicht nur im Reagenzglas, sondern auch in der lebenden Pflanze vollziehen, daß vor allem 
die aus den Blüten extrahierten Farbstoffe in der Tat die ihnen zugeschriebene Zusammensetzung haben.

Über diesen Weg muß die Genetik gehen, wenn sie die materielle Grundlage ihrer Faktoren finden will. Darum muß sie sich mit den chemischen Tatsachen bekannt machen, die die umfassende Arbeit Willstätters klargelegt hat und weiter klarlegt. Der methodische Teil dieser Arbeiten ist im Original einzusehen. Uns interessieren hier nur die Resultate, die im folgenden kurz zusammengefaßt werden sollen ${ }^{1}$ ):

1. Die aus den verschiedensten Pflanzen und Pflanzenteilen extrahierten Anthocyane erwiesen sich sämtlich als Glukoside. Cyanin z. B. = Cyanidin + 2 Mol. Glukose. (Abweichende Ansichten früherer Autoren sind mit demselben Resultat nachgeprüft worden.) In organischen LösungsmitteIn, Äther, Chloroform, Amylalkohol sind die Anthocyane als Glukoside unlöslich, die zuckerfreie Farbkomponente dagegen löslich; sie läßt sich daher nach der Hydrolyse quantitativ mit Amylalliohol ausschïtteln. Willstätter schlägt vor, das Glukosid als Cyanin, die Farbstoffkomponente als Cyanidin zu bezeichnen (in diesem Sinne soll die Nomenklatur weiterhin gebraucht werden). Die folgenden Reaktionen beziehen sich auf beide, Cyanin wie Cyanidin.

2. Die speziellen Untersuchungen wurden zunächst mit der Kornblume ausgeführt, die blau-, violett- und rotgefärbte Petalen besitzt. Das Material, mit dem die Pflanze arbeitet, ist verhältnismäßig einfach. Es ist nur ein Farbstoff vorhanden, aber in verschiedenen Modifikationen: violett als freie Säure (Cyanin), die ein Oxoniumphenolat darstellt, blau als Kaliumsalz des Cyanins, rot als Oxoniumsalz des Cyanins mit einer Pflanzensäure (die bei der Darstellung durch $\mathrm{HCl}$ ersetzt wurde). Die rote Modifikation wird also entstehen bei Anwesenheit überschüssiger Säure, indem diese mit der Sauerstoffbase ein Salz bildet, die blane bei stark alkalischem Zellsaft.

3. Cyanin und Cyanidin sind sehr starke Sauerstoffbasen; der salzbildende Sauerstoff ist vierwertig und chinoid gebunden; nur in dieser Bindung

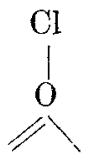

ist er farbbildend. Das Cyanin selbst ist ein inneres Salz der Oxoniumbase mit einem Phenylhydroxyl - die Anthocyanine sind also als Oxoniumphenolate anzusehen.

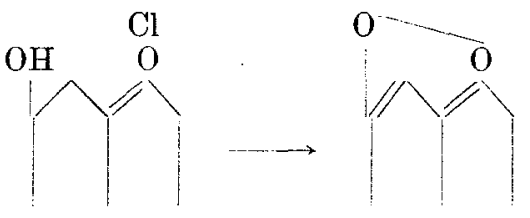

1) Über die Nomenklatur vgl. Anm. S. 90. 
4. Alle drei Verbindungen bilden farblose Isomere, die rote nur beim Fehlen überschüssiger Säure in wässeriger oder alkoholischer Lösung; bei Zusatz von Säure zu der farblosen Modifikation - unabhängig welcher Herkunft — regeneriert die Farbe zu rot.

5. Die Isomerisation zu einer farblosen Verbindung erklärt Willstätter durch Umwandlung des Farbsalzes in sein Carbinol unter Aufnahme eines Moleküls Wasser, wobei der chinoide Kern in einen aromatischen umgewandelt wird.

6. Die Isomerisation läßt sich durch Zusatz von $\mathrm{NaCl}$ und $\mathrm{NaNO}_{3}$ verhindern; wahrscheinlich bilden die Salze mit dem Cyanin haltbare Additionsprodukte. Dabei ist zu bemerken, daß die genannten Salze nicht osmotisch, sondern spezifisch wirken - sie sind z. B. durch $\mathrm{KCl}$ nicht zu ersetzen. Es ist anzunehmen, „daß in der Blüte der Farbstoff analog durch Bestandteile des Zellsaftes geschützt wird".

Die Anthocyanine zeigen so eine Reihe von Reaktionen, die auf ihre Verwandtschaft mit den Flavonen hinweisen, obwohl sich stets auch Differenzen zeigen.

a) Beide Gruppen sind Sauerstoffbasen und bilden mit Mineralsäuren Salze; da die Flavone nur schwache Basen sind, so werden ihre Salze schon durch Wasser zersetzt;

b) beide neigen zur Bildung farbloser Isomere; die der Cyanine sind ätherlöslich, wie die gelben Farbstoffe selbst;

c) Der empirischen Formel nach stehen die Anthocyanine den Flavonfarbstoffen sehr nahe; so ist das Cyanidin der Kornblume isomer u. a. mit Luteolin; das der Pellargonie mit dem Apigenin u. s. f. (52). Doch sind die Farbstoffe nicht die nächsten Verwandten ihrer Isomere aus der Flavongruppe, sondern derjenigen Flavone, die um ein Sauerstoffatom reicher sind, aber übereinstimmend substituierte Benzolreste besitzen. Aus diesen geh en sie durch Reduktion hervor; dabei kann die Reduktion je nach der Temperatur verschieden geleitet werden. Es ist hervorzuheben, daß die gewöhnlichen Reduktionsprodukte von Flavonen von den natürlichen BlütenAnthocyaninen verschieden sind. Willstätter führt dies auf eine Sprengung des Pyronringes zurück. Das wurde zunächst bei der Reduktion von Quercetin erkannt, das als erstes Produkt ein Allocyanidin, erst als Nebenprodukt das mit dem Farbstoff der Kornblume übereinstimmende Cyanidin lieferte.

Everest (51) hat eine ganze große Reihe von Extrakten gelber und weißer Blüten der Reduktion unterworfen und rote Lösungen erhalten. Sie sind jedoch noch nicht auf ihre Übereinstimmung mit den natürlichen Anthocyaninen verwandter Sippen untersucht werden, was Wheldale und Bassett mit Recht fordern, wenn die Entstehung der Anthocyanine auf diesem Wege aus den Anthoxanthinen hiernach allgemein gefolgert wird. Dagegen soll das Reduktionsprodukt des von Combes (41-43) aus Ampelopsisblättern 
isolierten gelben Farbstoffs mit dem aus dem roten Herbstlaub derselben Pflanze gewonnenen Anthocyanin identisch sein. Die Combessche Theorie über die Bildung der Anthocyanine durch Reduktion von Anthoxanthinen findet also durch Everest ihre Bestätigung.

Eine Verallgemeinerung ist indessen nach Wheldale und Bassett, insbesondere wegen des soeben gemachten Einwandes nicht zulässig. Auch eine Reihe anderer Bedenken läßt diese Forscher an der Oxydationshypothese festhalten: Die zwei voneinander durch die Höhe des Sauerstoffgehaltes unterschiedenen Anthocyanine, die Verff. aus Antirrhinum majus isolierten, unterscheiden sich von den Willstätterschen Blütenfarbstoffen einmal dadurch, daß sie keine Oxoniumsalze bilden; dann aber durch ihre bedeutendere Molekülgröße und ihren im Vergleich zu den bei Antirrhinum vorkommenden Anthoxanthinen größeren Sauerstoffgehalt; sind die Anthoxanthine die Chromogene der Anthocyanine, so muß Oxydation und Kondensation vor sich gegangen sein.

$\mathrm{Ob}$ in der Tat die hier untersuchten Blütenfarbstoffe - die in kristallisiertem Zustand noch nicht gewonnen sind - aus der großen Reihe der von Willstätter untersuchten und nach einheitlichem Plan aufgebanten rotviolett-blauen Farbstoffe herausgehoben werden können, bedarf der Nachprüfung.

Suchen wir zum Schluß uns zu vergegenwärtigen, an welchen Punkt die besprochenen Arbeiten die Mendelforschung geführt haben, so ergibt sich etwa folgendes Bild:

"Während einerseits einige Untersuchungen die Sicherheit ergeben haben, daß die genetischen Unterschiede der Blïtenfarben auch chemisch exakt faßbar sind, so ist andererseits deutlich, daß die als Arbeitshypothese von Miss Wheldale aufgestellte Oxydationshypothese nicht mehr die Forschungsrichtung in dieser Frage angeben kann. Drei Probleme werden damit besonders getroffen:

I. Der Zusammenhang zwischen den als Blütenfarbstoffe auftretenden Anthoxanthinen und Anthocyaninen ist durch diese rein chemischen Arbeiten nicht aufgedeckt worden. Selbst wenn der chemische Zusammenhang ob Reduktion, ob Oxydation - nicht noch strittig wäre, so bliebe immer noch der genetische Zusammenhang unerklärt. Warum z. B. bei Antirrhinum die Faktoren $\mathrm{B}$ und $\mathrm{C}$, die die gelbe Blütenfarbe bedingen, auch vorhanden sein müssen, damit ein Anthocyanin entstehen kann, bleibt eine offene Frage.

Vielleicht böte der folgend skizzierte Weg einige Aussicht auf Erfolg: Es müßten zwei Sippen untersucht werden, von denen jede einen Faktor für Blittenfarbe besitzt, ohne selbst gefärbt zu sein, und hieran anschließend als Kontrolle - oder vorausgehend als Wegweiser - der farbige Bastard beider. Sollte eine derartige Untersuchung nicht nur der Chemie, sondern gleichzeitig der Genetik zugute kommen, so müßte natürlich mit genetisch nicht nur einwandfrei reinem Material gearbeitet werden, sondern auch mit einer 
Pflanze, deren verschiedenste Sippen ihrer Erbformel nach bekannt sind wie Wheldales und Baurs Antirrhinum, oder Gregorys Primula, oder Bateson-Saunders Lathyrus, wobei die erstgenannte den Vorzug hat wegen der ausgesprochen rein auftretenden Anthoxanthine. Ein geeignetes Objekt wäre etwa eine elfenbeinfarbige Sippe von der Formel

$$
\text { BBCCffrr .... einerseits, }
$$

eine weiße von der Formel

bbccFFRR . . . anderseits,

deren Bastard von der Formal

\section{BbCcFfRr}

fleischfarben auf elfenbein wäre.

II. Ebenso aber können die erblich konstanten Abstufungen innerhalb der Farbreihen nicht einfach als verschiedene Oxydationsstufen angesehen werden; ihr chemischer Unterschied muß ergründet werden, wie der zwischen Anthoxanthinen und Anthocyaninen; aus dem Vorhandensein von Oxydasen auf ihr Eingreifen in den Färbungsproze $B \mathrm{zu}$ folgern, ist nicht zulässig.

III. Endlich ist auch auf diesem Wege die Natur des Hemmungsfaktors nicht zu bestimmen; den ohnehin sehr hypothetischèn Erklärungen, die auf S. 88 sub $B$ zusammengestellt sind, wird nach dem Fallen der Oxydationshypothese der Boden vollends entzogen.

Dagegen exöffnen die Resultate der Willstätterschen Arbeit eine neue Erklärungsmöglichkeit für das Zustandekommen rezessiv sowohl wie dominant weißer Blüten. Den Weg weisen die als Schutzstoffe gegen Isomerisation spezifisch wirkenden Salze $\mathrm{NaCl}$ und $\mathrm{NaNO}_{3}$ oder Konzentration von Wasserstoffionen. Es läßt sich wohl denken, daß die Gegenwart bzw. Abwesenheit derart wirkender Stoffe die "Erbfaktoren“ weißer Rassen ausmachen. Auch da käme es darauf hinaus, die Untersuchung an erbanalytisch bekanntem Material auszuführen.

Eine Frage von geringerer vererbungstheoretischer Bedeutung, die auch noch nicht einwandfrei beantwortet ist, betrifft die Spaltung des Glukosides bei der Farbbildung; sie kann bei Gelegenheit der andern Fragen mitgelöst werden.

Zum Schluß dieser Zusammenstellung soll noch einmal darauf hingewiesen werden, wie mühsam der Aufschluß über die wirklich kausalen Beziehungen beobachteter physiologischer Tatsachen ist. Da ist es erfreulich, wenn gerade von der Seite, von der die hypothetische Erklärung ausgegangen ist, die Notwendigkeit zu experimentieren und dadurch Material herbeizuschaffen, betont wird. Es ist fruchtlos über die Bedeutung der Erbfaktoren weiter zu spekulieren, ehe sich ihre Bildung in der Pflanze nicht vom chemischen Standpunkt übersehen läßt (Wheldale 47). 